\title{
An estimate of the time variation of the abundance gradient from planetary nebulae ${ }^{\star}$
}

\section{O, S, Ar, and Ne: a comparison of PN samples}

\author{
W. J. Maciel, L. G. Lago, and R. D. D. Costa
}

\author{
Instituto de Astronomia, Geofísica e Ciências Atmosféricas (IAG), Universidade de São Paulo, Rua do Matão 1226, \\ 05508-900 São Paulo SP, Brazil \\ e-mail: [maciel; leonardo; roberto]@astro.iag.usp.br
}

Received 21 November 2005 / Accepted 2 March 2006

\section{ABSTRACT}

\begin{abstract}
The time behaviour of the radial abundance gradients in the galactic disk is investigated on the basis of four different samples of planetary nebulae, comprising both smaller, homogeneous sets of data and larger, albeit non-homogeneous, samples. Four different chemical elements are considered, namely, oxygen, sulphur, argon, and neon. Our analysis supports our earlier conclusions that, on the average, the radial abundance gradients have flattened out in the last 6 to $8 \mathrm{Gyr}$.
\end{abstract}

Key words. planetary nebulae: general - Galaxy: abundances - Galaxy: evolution

\section{Introduction}

One of the most interesting observational properties that can be devised to constrain chemical evolution models is the magnitude, as well as the space and time variations, of the radial abundance gradients in the Galaxy (see for example Maciel \& Costa 2003; Maciel 2000; and Henry \& Worthey 1999, for recent reviews). Regarding the time variation in the gradients, which is possibly the most important information that can be obtained from the abundance variations in the galactic disk, the galactic subsystem of planetary nebulae $(\mathrm{PNe})$ plays a particularly important role. These objects have relatively well determined chemical compositions, which are based on the analysis of bright emission lines and are originated from stars within a reasonably large mass - and therefore age - bracket while on the main sequence. As a result, $\mathrm{PNe}$ can be used to investigate the possibility of a time variation of the average gradient in the galactic disk, in the same way as the open cluster stars (see for example Friel 1995; Friel et al. 2002).

In the first paper of this series, Maciel et al. (2003, hereafter referred to as Paper I) argued in favour of a time flattening of the $\mathrm{O} / \mathrm{H}$ gradient from roughly $-0.11 \mathrm{dex} / \mathrm{kpc}$ to $-0.06 \mathrm{dex} / \mathrm{kpc}$ during the last $9 \mathrm{Gyr}$, or from $-0.08 \mathrm{dex} / \mathrm{kpc}$ to $-0.06 \mathrm{dex} / \mathrm{kpc}$ in the last $5 \mathrm{Gyr}$, on the basis of a large sample of $\mathrm{PN}$ in the galactic disk. More recently, Maciel et al. (2005a, Paper II) extended the original discussion by (i) including $\mathrm{S} / \mathrm{H}$ data; (ii) adopting $[\mathrm{Fe} / \mathrm{H}] \times \mathrm{O} / \mathrm{H}$ and $[\mathrm{Fe} / \mathrm{H}] \times \mathrm{S} / \mathrm{H}$ conversions so that an estimate of the corresponding $[\mathrm{Fe} / \mathrm{H}]$ gradient could be made; (iii) estimating the average gradient from Cepheid data; and (iv) taking some recent determinations of the gradients from young objects into account, such as HII regions and stars in OB associations, and presenting a general comparison of the derived gradients of all the objects considered. It was shown in Paper II that the

* Based on observations made at the European Southern Observatory (Chile) and Laboratório Nacional de Astrofísica (Brazil). derived results lead to a consistent interpretation of a time flattening of the radial abundance gradients in the galactic disk, in agreement with the main results of Paper I.

In the present paper, we extend our previous results by taking two additional steps: (i) we consider four chemical elements in the discussion, namely oxygen, sulphur, argon, and neon, so that the ratios $\mathrm{O} / \mathrm{H}, \mathrm{S} / \mathrm{H}, \mathrm{Ar} / \mathrm{H}$, and $\mathrm{Ne} / \mathrm{H}$ can be studied; and (ii) we take four different PN samples into account, including three additional sets of data apart from the original abundances considered in Papers I and II. These samples are generally homogeneous sets, in the sense that all objects have been analysed by the same group under similar conditions, which includes data reduction and abundance determination. This is particularly important in order to make the previous results more robust, since all the large samples presently available are not homogeneous, having been compiled from a few individual homogeneous samples. As we will show in the next few sections, the general conclusions of Papers I and II are maintained.

\section{The samples}

\subsection{The basic sample}

The basic sample is essentially the same used in Papers I and II (Maciel et al. 2003, 2005a) and was described in detail in Paper I, to which the reader is referred. It is based on an earlier sample by Maciel \& Köppen (1994) and Maciel \& Quireza (1999), with some additional objects presented in Costa et al. (2004). This sample covers a wide range of galactocentric distances, roughly from $R=4$ to $R=14 \mathrm{kpc}$, with a stronger concentration of objects located up to $R=12 \mathrm{kpc}$. The main additions to our earlier sample are the anticentre objects from Costa et al. (2004), which are typically located at $R>10 \mathrm{kpc}$. Such a range is clearly wide enough to derive accurate gradients, and it in fact constitutes one of the strong points of using PN to study abundance variations in the galactic disk. 
Table 1. Number of PN in each sample.

\begin{tabular}{lrrrr}
\hline \hline Sample & $\mathrm{O} / \mathrm{H}$ & $\mathrm{S} / \mathrm{H}$ & $\mathrm{Ar} / \mathrm{H}$ & $\mathrm{Ne} / \mathrm{H}$ \\
\hline Basic Sample & 234 & 117 & 114 & 118 \\
Henry & 81 & 77 & 78 & 78 \\
Perinotto & 115 & 112 & 107 & 92 \\
IAG/USP & 71 & 58 & 62 & 18 \\
\hline
\end{tabular}

The total number of planetary nebulae for which reliable abundances of $\mathrm{O}, \mathrm{S}, \mathrm{Ar}$, and Ne have been obtained is shown in Table 1 for the basic sample, as well as for the remaining samples considered in this paper. These additional samples have approximately the same total galactocentric distance range as the basic sample, but they are smaller and more dispersed, with comparatively fewer objects at $R>10 \mathrm{kpc}$.

It can be seen that the basic sample is the largest one considered, but it should be kept in mind that it is a compilation, albeit careful, of several different determinations in the literature. Therefore, it lacks the homogeneity of the smaller available samples, so that it is interesting to investigate the extent to which the non-homogeneity degree affects the results of our analysis. Unfortunately, no sample is presently available that includes at the same time a high degree of homogeneity and a large enough size to be considered a complete sample - e.g. by including objects in the whole range of expected abundances.

The distances of the PN in the basic sample come from Maciel \& Quireza (1999) and Maciel \& Köppen (1994), and are basically from the statistical distance scale by Maciel (1984). For those nebulae not present in the catalogue of Maciel (1984), or those for which only distance limits were available, we have adopted values from more recent scales, especially Cahn et al. (1992; see the online material associated with Maciel \& Quireza 1999; and Table 1 of Costa et al. 2004). The same sources were used for the remaining samples. The problem of the distances to PN and their effect on the abundance gradients has been discussed elsewhere (see for example Maciel \& Köppen 1994). The analysis of large PN samples is necessarily based on statistical distances, which include a much larger number of objects than individually determined distances. Moreover, the uncertainties of the individual distances are often larger than generally assumed and, in many cases, not very different from those associated with the statistical distances. We may conclude that the use of different statistical distances may have a large influence on the distance of a particular object, but the global effect on a large sample is negligible, meaning the distance distribution is essentially unaffected. In other words, the abundance gradients are not noticeably affected, any more than their derived time variation. A recent study by Maciel \& Lago (2005) led to a similar conclusion regarding PN kinematics, which depends on the PN positions hence distances - on the galactic disk. It was found that the use of four different statistical distance scales (Maciel 1984; Cahn et al. 1992; van de Steene \& Zijlstra 1994; and Zhang 1995) for disk PN leads to a similar rotation curve, which is not very different from the Population I curve derived from HII regions (Clemens 1985).

\subsection{The Henry et al. sample (2004)}

The first homogeneous sample comes from the work of Henry and co-workers (Henry et al. 2004), who derived abundances of a set of 85 planetary nebulae based on observations at KPNO and CTIO. The detailed discussion of each step in the derivation is given in a series of previous papers (Kwitter \& Henry 2001;
Milingo et al. 2002a,b; and Kwitter et al. 2003). The abundances have been derived in a consistent and homogeneous manner and, especially, the sulphur abundances are expected to be particularly well-determined, as they have included near-IR lines of [SIII] at $\lambda 9069,9532 \AA$, apart from the optical lines of [SII] at $\lambda 6716,6731 \AA$ and [SIII] at $\lambda 6312 \AA$.

Some of the objects in the Henry et al. analysis are halo nebulae, which have been removed from our sample, as they are not adequate for studying disk properties such as abundance gradients.

Henry et al. (2004) have also analysed the existence of radial abundance gradients from their sample. Although no attempt has been made to investigate the time variation of the gradients, they were successful in deriving average gradients in the range -0.04 to $-0.05 \mathrm{dex} / \mathrm{kpc}$ for all studied elements, which are $\mathrm{O} / \mathrm{H}, \mathrm{Ne} / \mathrm{H}$, $\mathrm{S} / \mathrm{H}, \mathrm{Cl} / \mathrm{H}$, and $\mathrm{Ar} / \mathrm{H}$. Since their abundances - and to a large extent their distances - are completely independent of the sample considered in our previous papers, the fact that measurable and similar gradients have been detected is a nice confirmation of the usefulness of disk PNe as a chemical evolution tool.

\subsection{The Perinotto et al. sample (2004)}

Recently, Perinotto et al. (2004) presented a detailed analysis of the chemical composition of a large sample of planetary nebulae, for which they used the same extinction corrections, electron temperature, densities, and ionic abundance determinations, as well as ionization correction factors, including atomic constants and nebular models. Since the measured fluxes originated in different sources, this is not an entirely homogeneous sample, but its degree of homogeneity is clearly much higher than in the case of a simple compilation, in view of the rigorous criteria adopted for the inclusion of a given object and the same procedure applied to the measured fluxes. In fact, Perinotto et al. (2004) consider their sample as a highly homogeneous data set of galactic PN, determined with realistic uncertainties. Comparison of their abundances with some well-determined data in the literature, such as in the work by Kingsburgh \& Barlow (1994) generally produces a very good agreement. This sample is considerably larger, amounting to over 130 nebulae, some of which have been removed from our analysis since they may also belong to the halo or have no accurate distances.

\subsection{The IAG/USP sample}

The last sample considered in this paper comes from our own data, which we will call the IAG/USP sample. This is a highly homogeneous sample, although relatively small, reaching about 70 nebulae, after removing bulge objects and PN in the Magellanic Clouds. The observations have been made either at the $1.6 \mathrm{~m}$ LNA telescope in Brazil or with the $1.52 \mathrm{~m} \mathrm{ESO}$ telescope in Chile. In both cases, Boller and Chivens Cassegrain spectrographs were used. Details on the observations and reduction procedures can be found in the original papers (see for example Costa et al. 2004, and references therein).

\subsection{Comparison of $P N$ abundances}

A comparison of the abundances of the basic sample and the additional samples is shown in Figs. 1 to 4 for oxygen, sulphur, argon, and neon, respectively. For the sample by Henry et al. (2004), it can be seen that the oxygen, argon, and neon abundances generally show very good agreement with the basic 


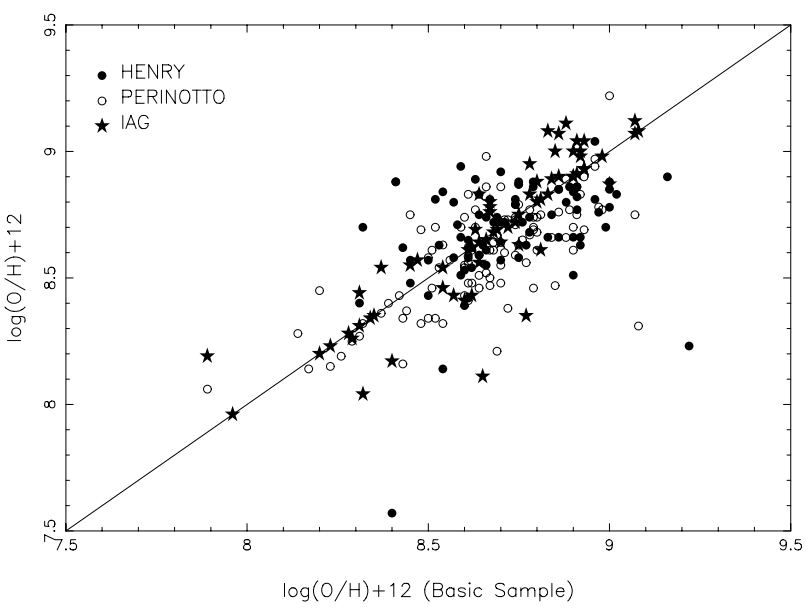

Fig. 1. A comparison of the $\mathrm{O} / \mathrm{H}$ abundances from the samples by Henry et al. (2004, filled circles), Perinotto et al. (2004, empty circles), and the IAG/USP sample (stars) with data from the basic sample.

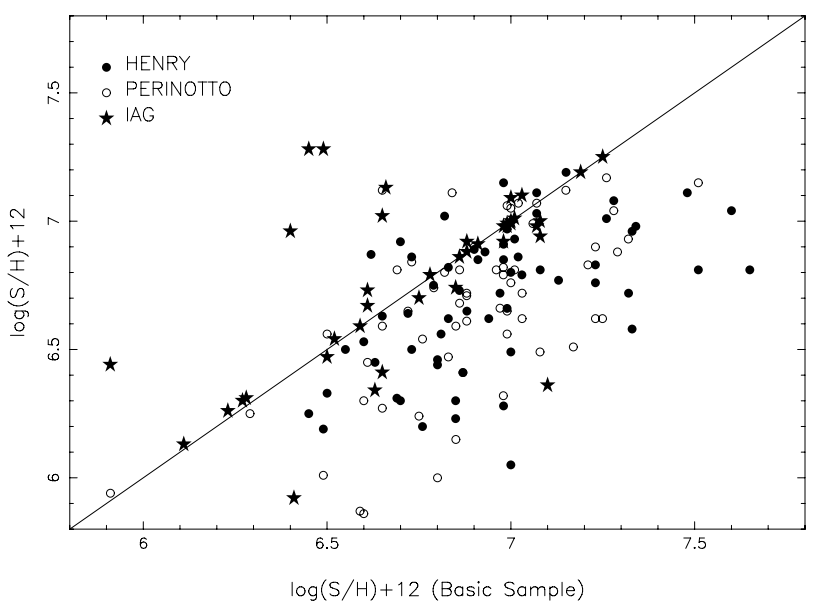

Fig. 2. The same as Fig. 1 for $\mathrm{S} / \mathrm{H}$.

sample, while part of the sulphur abundances shows some underabundance compared to the basic sample. In fact, the lower sulphur abundances were noticed by Henry et al. (2004) when making comparisons with HII region data, and was referred to as the "sulphur anomaly". Several reasons have been suggested to explain such a discrepancy (see the discussion by Henry et al.), to which we could add that their [SIII] electron temperatures are systematically higher than the corresponding [OIII] temperatures, which tends to decrease the derived sulphur abundances. A few outliers can be observed in Figs. 1 to 4 , and it should be noticed that some of them, such as $\mathrm{Hb} 12$ and IC 418, have also been identified as outliers in the plots presented by Henry et al. (2004).

The sample by Perinotto et al. (2004) also shows generally good agreement with the data in the basic sample, especially for oxygen (Fig. 1) and neon (Fig. 4). Their sulphur abundances and, to a lesser extent, argon are generally lower than in the basic sample (cf. Figs. 2 and 3) and show the same sulphur anomaly as discussed by Henry et al. (2004).

The smaller IAG sample (stars) shows several nebulae directly on the straight line of Figs. 1 to 4 , which reflects the fact that we have adopted several of the results of this sample in our merged basic sample. An exception is neon, for which our own data is very limited, as can be seen from Fig. 4 or Table 1 .

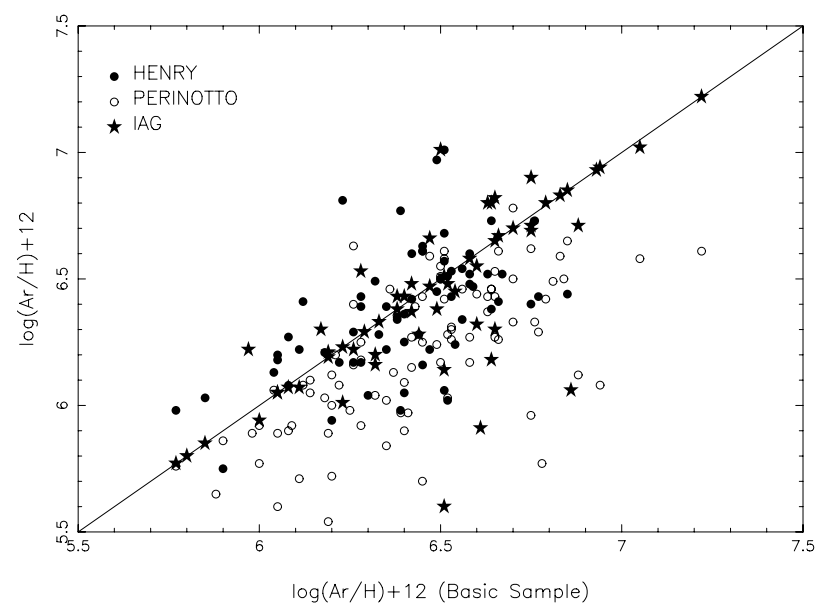

Fig. 3. The same as Fig. 1 for $\mathrm{Ar} / \mathrm{H}$.

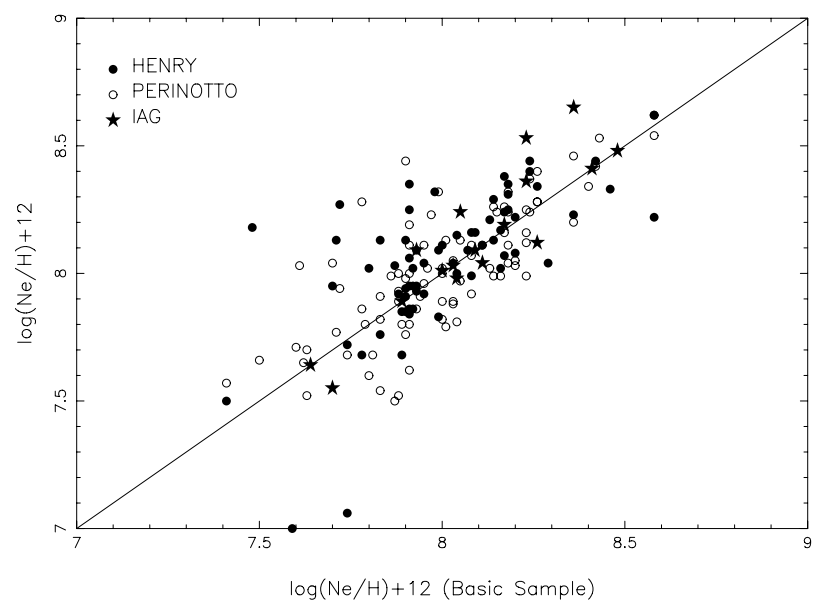

Fig. 4. The same as Fig. 1 for $\mathrm{Ne} / \mathrm{H}$.

\section{The method}

A detailed discussion of the method used in order to estimate the abundance gradients at different epochs is given in Papers I and II (Maciel et al. 2003, 2005a) to which the reader is referred for details. Basically, the abundance gradients in the form $\mathrm{d} \log (\mathrm{X} / \mathrm{H}) / \mathrm{d} R(\mathrm{dex} / \mathrm{kpc})$ were determined for $\mathrm{X}=\mathrm{O}, \mathrm{S}, \mathrm{Ar}$, and $\mathrm{Ne}$ assuming a linear variation in the abundances with the galactocentric distance $R$, namely, neglecting possible space variations within the galactic disk of the gradients themselves. A value of $R_{0}=8.0 \mathrm{kpc}$ was adopted for the position of the LSR, as in the previous papers.

The samples were divided into age groups, and the ages were estimated in the following way. First, the heavy element abundances, that is, $\mathrm{O} / \mathrm{H}, \mathrm{S} / \mathrm{H}, \mathrm{Ar} / \mathrm{H}$, and $\mathrm{Ne} / \mathrm{H}$, were converted into $[\mathrm{Fe} / \mathrm{H}]$ metallicities using a well-determined $[\mathrm{O} / \mathrm{H}] \times[\mathrm{Fe} / \mathrm{H}]$ relationship for the galactic disc, as well as correlations of $\mathrm{S} / \mathrm{H}$, $\mathrm{Ar} / \mathrm{H}$, and $\mathrm{Ne} / \mathrm{H}$ with the oxygen abundance $\mathrm{O} / \mathrm{H}$. These correlations have been studied by several people (see for example Henry et al. 2004), and the slopes are very close to unity, reflecting that, as a first approximation, all four element ratios are good tracers of the interstellar abundances at the time of the formation of the PN progenitors. From the $[\mathrm{Fe} / \mathrm{H}]$ metallicities, the ages were determined using an age-metallicity relationship (AMR) by Edvardsson et al. (1993), which also depends on the galactocentric distance. Such a relationship constitutes an improvement over to the solar neighbourhood AMR generally 


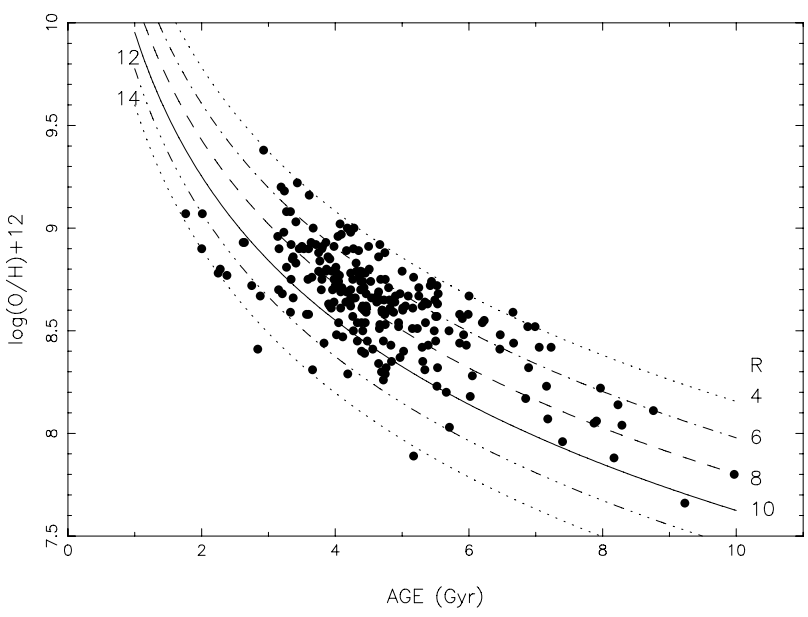

Fig. 5. The adopted age-metallicity relation from Edvardsson et al. (1993) which depends on the galactocentric distance $R$. The AMR are plotted for $\mathrm{O} / \mathrm{H}$ abundances and galactocentric distances in the range $4<R(\mathrm{kpc})<14$. The dots show the PN of the basic sample.

adopted, although it should be considered as an approximation when applied to the galactic disk. As an example, Fig. 5 shows the adopted age-metallicity relation as applied to the $\mathrm{O} / \mathrm{H}$ abundances of the PN in the basic sample. It can be seen that the whole age bracket extends from $1 \mathrm{Gyr}$ to $10 \mathrm{Gyr}$, approximately, with a higher concentration of objects with ages around 4 to 5 Gyr.

The adoption of an age-metallicity relation is probably our main source of uncertainty, even allowing for some galactocentric dependence. Although most average AMR in the literature are similar to each other (see for instance Rocha-Pinto et al. 2000), there is still an ongoing discussion of their intrinsic dispersion at any given age. An alternative approach involves directly determining the central star masses, hence ages, from the nebular chemical composition, as we did in Paper I, with similar results for the time variation in the abundance gradients. Such a procedure can also be applied to the predictions of theoretical models of intermediate mass stars. As an example, we recently considered models by Marigo (2001) and our preliminary results (Maciel et al., in preparation) largely support the results based on the age-metallicity relation.

Once the individual ages had been determined, the nebulae in each sample were divided into two age groups, one called the younger Group I and the other the older Group II. As in Paper II, if $t_{\mathrm{I}}$ is the upper age limit of Group I, all PN progenitors having ages $t \leq t_{\mathrm{I}}$ will belong to Group I, while those with ages $t>t_{\mathrm{I}}$ belong to Group II. Several values of $t_{\mathrm{I}}$ have been considered in the range $3.0<t_{\mathrm{I}}(\mathrm{Gyr})<6.0$, and for each of these values we calculated the gradients of Groups I and II and the corresponding correlation coefficients. Therefore, our conclusions do not depend on the adopted age limit $t_{\mathrm{I}}$; that is, they are independent of the detailed manner by which the groups are defined. Of course, for values of the age limit very close to the limiting values of the adopted range, namely $t_{\mathrm{I}} \simeq 3$ or $6 \mathrm{Gyr}$, either Group I or Group II becomes underpopulated, so that the corresponding results are less reliable (see Paper II for a detailed discussion).

It should be stressed that the main goal of this project is to establish the time variation for the abundance gradients, that is, to determine whether the gradients steepen or flatten out with time. Therefore, we are not particularly concerned with an accurate determination of the absolute magnitude of the gradients at any given epoch.

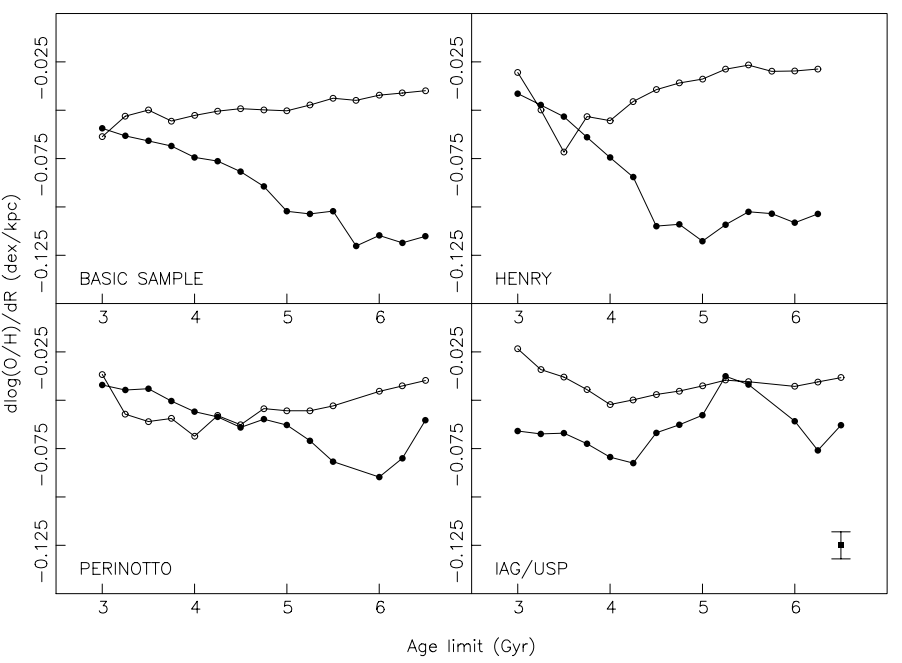

Fig. 6. Time variation in the $\mathrm{O} / \mathrm{H}$ gradient from $\mathrm{PNe}$. The four samples were divided into two age groups, Group I ("younger"), with ages lower than the age limit $t_{\mathrm{I}}$, and Group II ("older"), with ages higher than $t_{\mathrm{I}}$. The plot shows the $\mathrm{O} / \mathrm{H}$ gradient $(\mathrm{dex} / \mathrm{kpc})$ of each group as a function of the upper age limit of Group I, $t_{\mathrm{I}}$, for the four samples considered. The gradients of the younger Group I (empty circles connected by lines) are flatter than those of the older Group II (filled circles connected by lines). An average error bar is given in the lower right corner of the last panel.

\section{Results and discussion}

\section{1. $\mathrm{O} / \mathrm{H}$}

The main results of the time variation in the abundance gradients of the $\mathrm{O} / \mathrm{H}$ ratio are shown in Fig. 6, where we plot the derived gradients as a function of the adopted age limit $t_{\mathrm{I}}$. These are the best results of all four elements, as the average $\mathrm{O} / \mathrm{H}$ abundances are higher and better determined and the samples are larger. From Fig. 6, it can be concluded that the gradients of the younger Group I are systematically flatter than the corresponding gradients of the older Group II, supporting our earlier conclusions in Papers I and II. These results apply to all four samples considered, especially the larger basic sample, the sample by Henry et al. (2004), and the IAG/USP sample. For the basic sample (top left panel), the results are essentially the same as presented in Fig. 1 of Paper II.

In the case of the sample by Perinotto et al. (2004, bottom left panel), the gradients of both groups are similar for age limits in the range $3<t_{\mathrm{I}}(\mathrm{Gyr})<4.5$, but for larger age limits the separation of the two groups is clear.

In view of the comment at the end of Sect. 3, the younger Group I is underpopulated for $t_{\mathrm{I}} \simeq 3 \mathrm{Gyr}$, and the same occurs at $t_{\mathrm{I}} \simeq 6 \mathrm{Gyr}$ for Group II. Therefore, the statistical results are less reliable near these limiting values, as the space distribution of the nebulae may not be homogeneous. We would then expect the best results to be associated with groups containing a reasonably large fraction of the whole sample. As an illustration, the age limit at which both groups have the same number of elements (that is, half of the sample) occurs typically for $3.9<t_{\mathrm{I}}(\mathrm{Gyr})<4.6$, showing no systematic differences between the space distributions of the PN groups.

A detailed discussion of the errors involved in the determination of the gradients, as well as a statistical analysis of the different object samples considered were recently given by Maciel et al. (2005b). For the sake of completeness, we have included in the lower right corner of the last panel of Fig. 6 an average error 


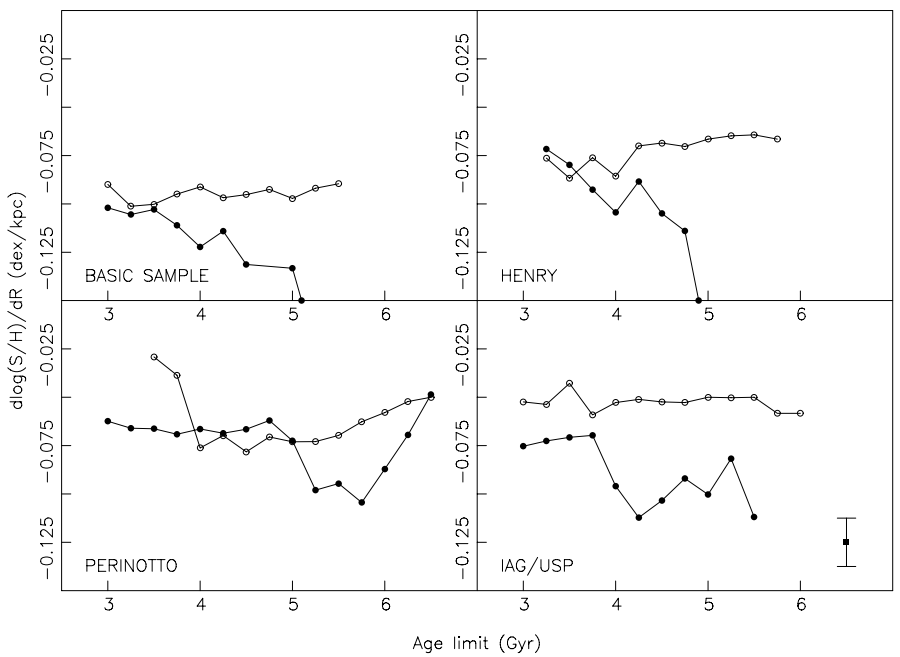

Fig. 7. The same as Fig. 6 for $\mathrm{S} / \mathrm{H}$.

bar concerning the $\mathrm{O} / \mathrm{H}$ gradient from $\mathrm{PN}$. The same procedure has been adopted in the corresponding figures of the other element ratios, namely $\mathrm{S} / \mathrm{H}, \mathrm{Ar} / \mathrm{H}$, and $\mathrm{Ne} / \mathrm{H}$.

Another important quantity that can be used in the interpretation of Fig. 6 is the correlation coefficient of the linear fits. For $\mathrm{O} / \mathrm{H}$, the coefficients of all fits shown in Fig. 6 are large, typically in the range $0.50<|r|<0.90$, so that the flattening of the gradients of the younger Group I relative to the older Group II is real.

\section{2. $S / H$}

For the $\mathrm{S} / \mathrm{H}$ ratio, the same behaviour of the $\mathrm{O} / \mathrm{H}$ gradients is observed, as can be seen from Fig. 7. This is true for the basic sample, the sample by Henry et al. (2004), and the IAG/USP sample. Again the gradients of the younger Group I are systematically flatter than for the older Group II, and the correlation coefficients are also large, in the range $0.50<|r|<0.80$. For the basic sample, the correlation coefficients of both groups are large, $0.70<|r|<0.80$. The results of the top left panel are also essentially the same as in Fig. 2 of Paper II. For these three samples, the average gradients of Group I are independent of the adopted age limit, while the corresponding gradients of the older groups are increasingly steep, although the derived values for age limits close to the upper limit of $6 \mathrm{Gyr}$ are less reliable, as mentioned.

It is interesting to notice that the behaviour of $\mathrm{S} / \mathrm{H}$ gradients of the basic sample and the Henry et al. sample are very similar, as shown by the top panels of Fig. 7. As mentioned in Sect. 2, the sulphur abundances of the sample by Henry et al. are expected to be very accurate, further confirming the flattening of the gradients for the younger Group I. It can also be concluded that the "sulphur anomaly" present in the Henry et al. sample does not affect the picture of time evolution for the gradients as derived from the basic sample.

In the sample by Perinotto et al. (2004), S/H shows a similar behaviour as $\mathrm{O} / \mathrm{H}$, in the sense that the gradients of both groups are of the same order of magnitude for lower values of the age limit, $t_{\mathrm{I}}<4.5 \mathrm{Gyr}$, presenting some flattening of the younger Group I at higher values of $t_{\mathrm{I}}$. For this sample, the correlation coefficients reach the lowest values, typically $|r| \simeq 0.4$ to 0.5 . Again for illustration purposes, in all cases of Fig. 7, both Groups I and II are approximately the same size for $4.1<t_{\mathrm{I}}(\mathrm{Gyr})<4.6$.

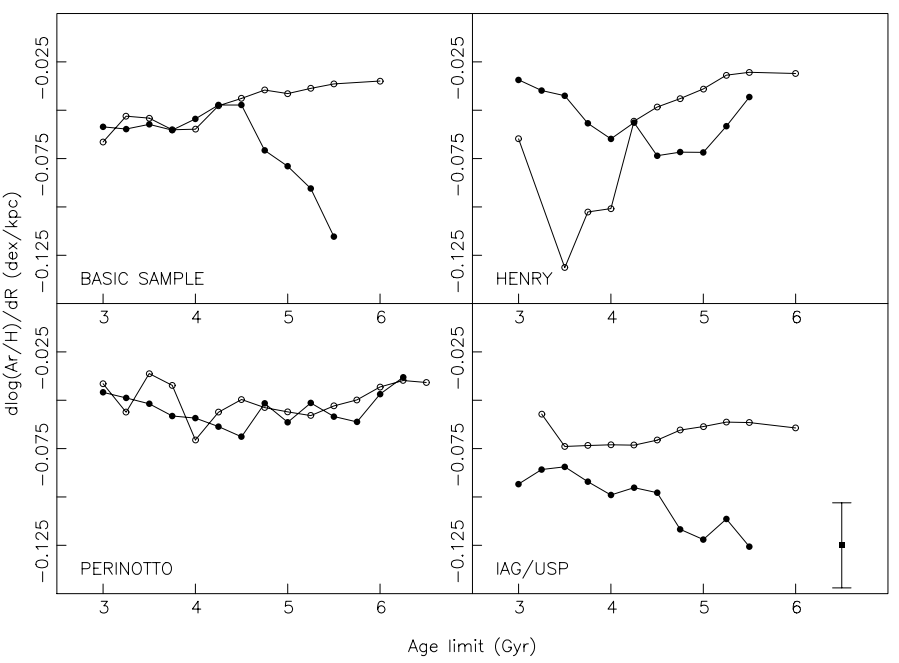

Fig. 8. The same as Fig. 6 for $\mathrm{Ar} / \mathrm{H}$.

\section{3. $\mathrm{Ar} / \mathrm{H}$}

The Ar/H gradients shown in Fig. 8 also indicate some flattening of the younger Group I in the basic sample, especially for age limits $t_{\mathrm{I}}>4.5$ Gyr. The corresponding correlation coefficients are moderate, typically $|r| \simeq 0.50$.

For the IAG/USP sample, the flattening is more evident, as can be seen from the bottom right panel of Fig. 8, where Group I indicates less pronounced gradients than Group II. Here the correlation coefficients are in the range $0.50<|r|<0.80$.

In the sample by Henry et al., some flattening of Group I can also be noticed for $t_{\mathrm{I}}>4 \mathrm{Gyr}$. In all panels of Fig. 8 both groups have similar sizes for $3.8<t_{\mathrm{I}}(\mathrm{Gyr})<4.6$, so that the observed flattening is probably real. However, the correlation coefficients in this range are modest, typically $|r| \simeq 0.40$ for Group I and somewhat higher $(|r| \simeq 0.60)$ for Group II, so that this conclusion cannot be stressed too much.

For this element, the sample by Perinotto et al. does not lead to any definite conclusions. In this case, the gradients of both groups are very similar, within the expected uncertainties of the gradients themselves, which are typically $0.02 \mathrm{dex} / \mathrm{kpc}$, as can be seen from Papers I and II. Since the correlation coefficients are modest $(|r| \simeq 0.4$ to 0.6 ), we should probably conclude that no differences can be observed between the gradients of the different groups.

\section{4. $\mathrm{Ne} / \mathrm{H}$}

Finally, Fig. 9 shows the results of the Ne/H ratio for our adopted samples. We can see that a well defined flattening of the younger Group I compared to the older Group II is apparent for the Henry et al. (2004) sample, for which the correlation coefficients are also moderate to large, being in the range $0.40<|r|<0.60$ for Group I and $0.50<|r|<0.80$ for Group II. Such a result is particularly important, as the remaining two samples do not allow clear conclusions to be drawn. For this sample, both groups have approximately the same size for $t_{\mathrm{I}} \simeq 4.3 \mathrm{Gyr}$.

The Perinotto et al. sample displays similar gradients for both age groups. The correlation coefficients are typically in the range $0.50<|r|<0.80$, so that we conclude from this sample that the gradients of both groups are similar.

In the case of the basic sample, acceptable gradients can only be obtained for the younger Group I, for which the correlation coefficients are well defined and typically in the range 


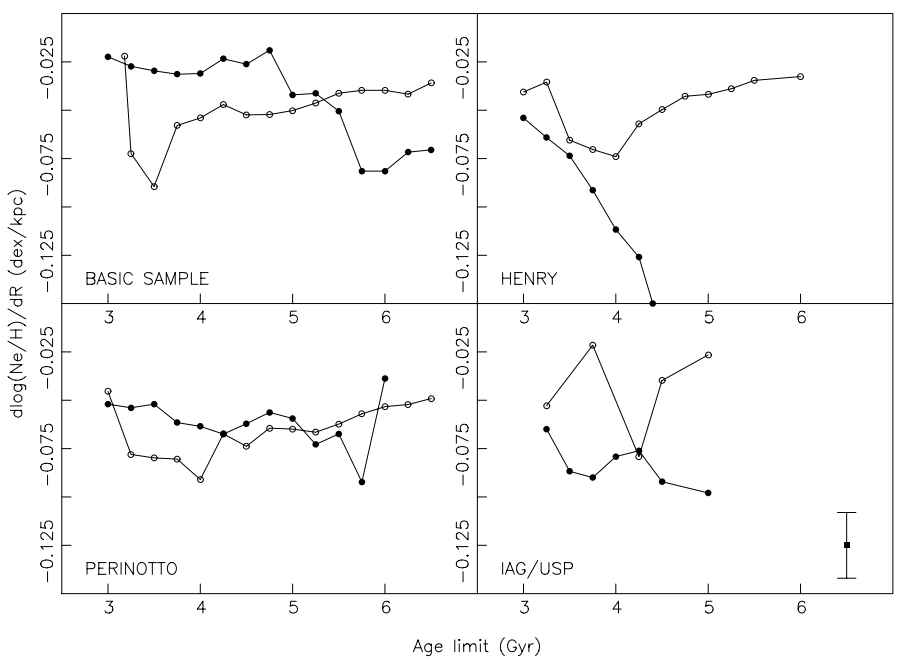

Fig. 9. The same as Fig. 6 for $\mathrm{Ne} / \mathrm{H}$.

$0.40<|r|<0.80$, so that the derived gradients are probably correct. However, for the older groups the gradient is very flat for most of the range in age limits, and the correlation coefficients are tipically $|r|<0.20$, which is consistent with the very low gradients displayed by this group. This means that the gradients are not well defined in this group, so that no comparison of the time evolution of the gradients can be made.

As mentioned before, the IAG/USP sample includes only a very limited number of $\mathrm{PN}$ with well-determined $\mathrm{Ne} / \mathrm{H}$ abundances, so that no reliable gradients can be obtained from this sample. The corresponding panel in Fig. 9 shows some indication of a flattening for the younger Group I, but the correlations are rather poor and the sample is very small. The gradients of the older Group II are better, so that we can conclude that the results of this sample are consistent with the results of the sample by Henry et al. It seems that for this element the homogeneity of the sample is especially important, as the less homogeneous samples (the basic and the Perinotto samples) present uncertain and even conflicting results, while the the homogeneous sample by Henry et al. (1999) shows very clear results.

\section{Time evolution of the $[\mathrm{Fe} / \mathrm{H}]$ gradient}

Taken together, the results shown in Figs. 6 to 9 generally confirm the main conclusions of Papers I and II, in the sense that there is a clear tendency for the gradients to flatten out, at least during the lifetimes of the older objects considered here. When adopting 13.6 Gyr for the age of the Galaxy, as in the previous papers, the gradients have been flattening out in the last 6 to $8 \mathrm{Gyr}$, approximately, or since the Galaxy was approximately 6 Gyr old. From the data considered here, as in the previous papers, nothing can be said about the time evolution of the abundance gradients during the early epochs of galactic evolution, that is, for $t \leq 6$ Gyr. These results cannot be taken as definitive, in view of the uncertainties and assumptions considered, especially regarding the abundances and age determinations. However, as we see below, we have considerably expanded our original analysis to include new elements, samples, and galactic objects, so that our conclusions are now based on a more solid foundation.

The inclusion of $\mathrm{Ar}$ and $\mathrm{Ne}$ generally supports the earlier conclusions drawn from $\mathrm{O} / \mathrm{H}$ (Paper I) and S/H (Paper II), although these elements are clearly more limited in view of

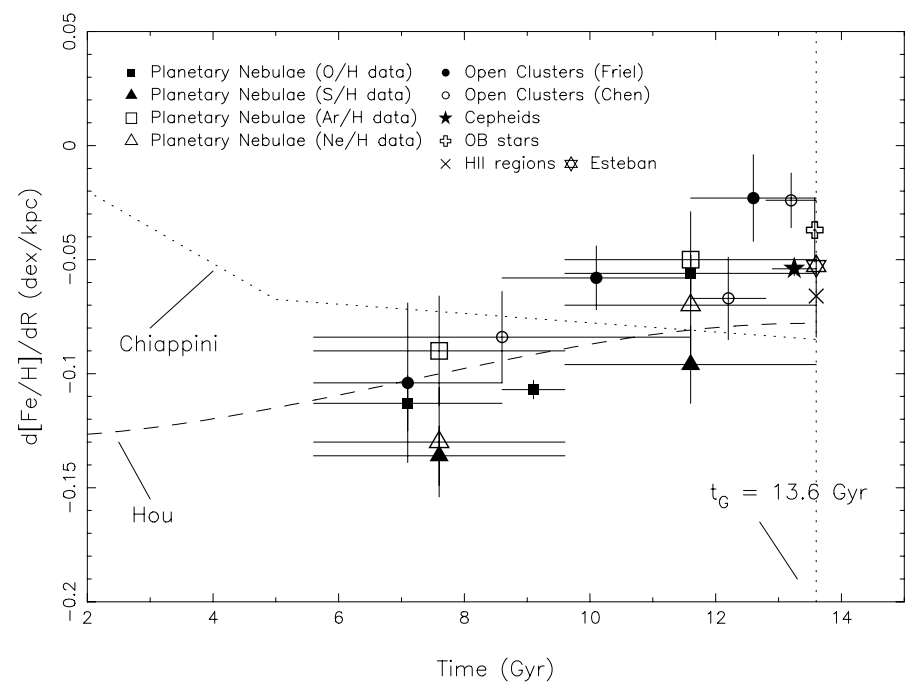

Fig. 10. Time variation of the $[\mathrm{Fe} / \mathrm{H}]$ abundance gradient $(\mathrm{dex} / \mathrm{kpc})$, as in Fig. 8 from Paper II, with the following additions: (i) converted $[\mathrm{Fe} / \mathrm{H}]$ gradients from $\mathrm{Ar} / \mathrm{H}$ abundances in $\mathrm{PN}$ analyzed in the present paper (empty squares); (ii) the same for $\mathrm{Ne} / \mathrm{H}$ gradients (open triangles); (iii) recent results by Esteban et al. (2005) for HII regions (star of David); and (iv) theoretical tracks from Chiappini et al. (2001, dotted line). The remaining data are: (i) $[\mathrm{Fe} / \mathrm{H}]$ gradients from $\mathrm{PN}$ calculated from $\mathrm{O} / \mathrm{H}$ data (filled squares); (ii) the same for $\mathrm{S} / \mathrm{H}$ (filled triangles); (iii) Open cluster data from Friel et al. (2002, filled circles); (iv) the same from Chen et al. (2003, empty circles); (v) Cepheid data (filled star); (vi) OB stars in associations (thick cross); (vii) HII regions from the literature ( $\times$ sign); and (viii) theoretical models by Hou et al. (2000, dashed line). The dotted vertical line shows the adopted age of the galactic disk, $t_{\mathrm{G}}=13.6 \mathrm{Gyr}$.

the lower, more uncertain abundances, and the smaller samples. In this respect, the homogeneous sample by Henry et al. (2004) is particularly useful, especially for neon. Adopting this sample as representative of the the $\mathrm{Ne} / \mathrm{H}$ gradient, we would estimate for Group I (ages between 0 and $4 \mathrm{Gyr}$ ) an average gradient of $\mathrm{d} \log (\mathrm{Ne} / \mathrm{H}) / \mathrm{d} R \simeq-0.06 \mathrm{dex} / \mathrm{kpc}$, and for Group II (ages between 4 and 8 Gyr) an average gradient of $\mathrm{d} \log (\mathrm{Ne} / \mathrm{H}) / \mathrm{d} R \simeq-0.11 \mathrm{dex} / \mathrm{kpc}$, which would approximately correspond to an $[\mathrm{Fe} / \mathrm{H}]$ gradient $\mathrm{d}[\mathrm{Fe} / \mathrm{H}] / \mathrm{d} R \simeq-0.07 \mathrm{dex} / \mathrm{kpc}$ and $\mathrm{d}[\mathrm{Fe} / \mathrm{H}] / \mathrm{d} R \simeq-0.13 \mathrm{dex} / \mathrm{kpc}$ for Groups I and II, respectively (see Paper II for details in the conversion of observed abundance gradients to $[\mathrm{Fe} / \mathrm{H}]$ gradients).

For $\mathrm{Ar} / \mathrm{H}$, using the same procedure and taking the basic sample into account, as well as the sample by Henry et al. (2004), we would obtain $\mathrm{d} \log (\mathrm{Ar} / \mathrm{H}) / \mathrm{d} R \simeq-0.04 \mathrm{dex} / \mathrm{kpc}$ or $\mathrm{d}[\mathrm{Fe} / \mathrm{H}] / \mathrm{d} R \simeq-0.05 \mathrm{dex} / \mathrm{kpc}$ for Group I and $\mathrm{d} \log (\mathrm{Ar} / \mathrm{H}) / \mathrm{d} R \simeq$ $-0.075 \mathrm{dex} / \mathrm{kpc}$ or $\mathrm{d}[\mathrm{Fe} / \mathrm{H}] / \mathrm{d} R \simeq-0.09 \mathrm{dex} / \mathrm{kpc}$ for Group II.

These estimates are plotted in Fig. 10, which reproduces Fig. 8 of Paper II with the following additions: (i) the Ar/H gradients from $\mathrm{PN}$ converted into $[\mathrm{Fe} / \mathrm{H}]$ gradients are included, as described above; (ii) the same for $\mathrm{Ne} / \mathrm{H}$ gradients; (iii) recent results by Esteban et al. (2005) for $\mathrm{O} / \mathrm{H}$ gradients in $\mathrm{HII}$ regions based on abundances derived from recombination lines, converted into $[\mathrm{Fe} / \mathrm{H}]$ gradients as in Paper II; and (iv) predictions of theoretical models by Chiappini et al. (2001). The remaining results plotted in Fig. 10 are the same as in Fig. 8 of Paper II, namely: (i) $\mathrm{O} / \mathrm{H}$ data from $\mathrm{PN}$ converted into [Fe/H] gradients, considering three age groups (young, intermediate, old); (ii) the same for $\mathrm{S} / \mathrm{H}$; (iii) $[\mathrm{Fe} / \mathrm{H}]$ data from open clusters from the sample by Friel et al. (2002); (iv) the same for the sample by Chen et al. (2003); (v) $[\mathrm{Fe} / \mathrm{H}]$ data from cepheids 
(Andrievsky et al. 2002a,b,c, 2004; Luck et al. 2003); (vi) OB stars and associations from Daflon \& Cunha (2004); and (vii) HII region data from the recent literature, as discussed in Paper II. Finally, predictions by theoretical models by Hou et al. (2000) can be compared with the previously mentioned results by Chiappini et al. (2001) as illustrations of theoretical models of chemical evolution.

As mentioned in Paper II, our purpose here is to provide observational constraints to chemical evolution models, rather than to discuss the different models available in the literature in detail. Nevertheless, it is interesting to comment on the large discrepancy of the models by Hou et al. (2000) and Chiappini et al. (2001) as observed in Fig. 10, especially at earlier epochs of the galactic lifetime. Hou et al. (2000) adopt an exponentially decreasing infall rate and an "inside-out" scheme for the formation of the galactic disk, in which a rapid increase in the metal abundance at early times in the inner disk leads to a steep gradient. As the star formation migrates to the outer disk, metal abundances are enhanced in that region, so that the gradients flatten out. Similar results have also been obtained by Alibés et al. (2001), among others. In the models by Chiappini et al. (2001), two infall episodes are assumed to form the halo and the disk. The latter also has an "inside-out" formation scenario, in which the timescale is a linear function of the galactocentric distance. The detailed time variation of the gradients depends on the particular model, but in general some steepening of the gradients is predicted, which does not seem to be supported by the data presented in Fig. 10. Possibly, the different timescales for star formation and infall in the models by Hou et al. (2000) and Chiappini et al. (2001) are responsible for their different predictions of the time variation in the abundance gradients.

It can also be seen that the new results fit the picture previously sketched in Paper II nicely, so that the same average flattening rate can be advocated here, namely, $\mathrm{d}[\mathrm{Fe} / \mathrm{H}] / \mathrm{d} R \sim 0.005$ to $-0.010 \mathrm{dex} \mathrm{kpc}^{-1} \mathrm{Gyr}^{-1}$ for the last 6 to $8 \mathrm{Gyr}$. This value refers to a spatially-averaged gradient, corresponding to a linear variation in the abundances with the galactocentric distance. In practice, the situation is more complicated, and all quoted theoretical models predict some space variations of the gradients along with their temporal variations. Since the observational results on the space variations are still very limited and often contradictory, further work is needed in order to definitely establish the characteristics of this important observational constraint.
Acknowledgements. This work was partly supported by FAPESP (grant 02/08816-5), CNPq and CAPES. Observations at ESO/Chile were possible through the FAPESP grant 98/10138-8.

\section{References}

Alibés, A., Labay, J., \& Canal, R. 2001, A\&A, 370, 1103

Andrievsky, S. M., Bersier, D., Kovtyukh, V. V., et al. 2002b, A\&A, 384, 140

Andrievsky, S. M., Kovtyukh, V. V., Luck, R. E., et al. 2002a, A\&A, 381, 32

Andrievsky, S. M., Kovtyukh, V. V., Luck, R. E., et al. 2002c, A\&A, 392, 491

Andrievsky, S. M., Luck, R. E., Martin, P., \& Lépine, J. R. D. 2004, A\&A, 413, 159

Cahn, J. H., Kaler, J. B., \& Stanghellini, L. 1992, A\&AS, 94, 399

Chen, L., Hou, J. L., \& Wang, J. J. 2003, AJ, 125, 1397

Chiappini, C., Matteucci, F., \& Romano, D. 2001, ApJ, 554, 1044

Clemens, D. P. 1985, ApJ, 295, 422

Costa, R. D. D., Uchida, M. M. M., \& Maciel, W. J. 2004, A\&A, 423, 199

Daflon, S., \& Cunha, K. 2004, ApJ, 617, 1115

Esteban, C., Garcia-Rojas, J., Peimbert, M., et al. 2005, ApJ, 618, L98

Edvardsson, B., Andersen, J., Gustafsson, B., et al. 1993, A\&A, 275, 101

Friel, E. D. 1995, ARA\&A, 33, 381

Friel, E. D., Janes, K. A., Tavarez, M., et al. 2002, AJ, 124, 2693

Henry, R. B. C., Kwitter, K. B., \& Balick, B. 2004, AJ, 127, 2284

Henry, R. B. C., \& Worthey, G. 1999, PASP, 111, 919

Hou, J. L., Prantzos, N., \& Boissier, S. 2000, A\&A, 362, 921

Kingsburgh, R. L., \& Barlow, M. J. 1994, MNRAS, 271, 257

Kwitter, K. B., \& Henry, R. B. C. 2001, ApJ, 562, 804

Kwitter, K. B., Henry, R. B. C., \& Milingo, J. B. 2003, PASP, 115, 80

Luck, R. E., Gieren, W. P., Andrievsky, S. M., et al. 2003, A\&A, 401, 939

Maciel, W. J. 1984, A\&AS, 55, 253

Maciel, W. J. 2000, in The Evolution of the Milky Way, ed. F. Matteucci, \& F. Giovannelli (Dordrecht: Kluwer), 81

Maciel, W. J., \& Costa, R. D. D. 2003, Planetary Nebulae, ed. S. Kwok, M. Dopita, \& R. Sutherland (San Francisco: ASP), IAU Symp., 209, 551

Maciel, W. J., Costa, R. D. D., \& Uchida, M. M. M. 2003, A\&A, 397, 667 (Paper I)

Maciel, W. J., \& Köppen, J. 1994, A\&A, 282, 436

Maciel, W. J., \& Lago, L. G. 2005, Rev. Mex. Astron. Astrofis., 41, 383

Maciel, W. J., Lago, L. G., \& Costa, R. D. D. 2005a, A\&A, 433, 127 (Paper II)

Maciel, W. J., Lago, L. G., \& Costa, R. D. D. 2005b, Planetary Nebulae as Astronomical Tools, ed. R. Szczerba, G. Stasińska, \& S. K. Górny (AIP), 246

Maciel, W. J., \& Quireza, C. 1999, A\&A, 345, 629

Marigo, P. 2001, A\&A, 370, 194

Milingo, J. B., Henry, R. B. C., \& Kwitter, K. B. 2002a, ApJS, 138285

Milingo, J. B., Kwitter, K. B., Henry, R. B. C., \& Cohen, R. E. 2002b, ApJS, 138 279

Perinotto, M., Morbidelli, L., \& Scatarzi, A. 2004, MNRAS, 349, 793

Rocha-Pinto, H. J., Maciel, W. J., Scalo, J., \& Flynn, C. 2000, A\&A, 358, 850

van de Steene, G., \& Zijlstra, A. 1994, A\&AS, 108, 485

Zhang, C. Y. 1995, ApJS, 98, 659 